\title{
Effects of Hall Current and Ohmic Heating on Non-Newtonian Fluid Flow in a Channel due to Peristaltic Wave
}

\author{
Md. Maruf Hasan 1,2, Md. Abdus Samad², Md. Mubarak Hossain² \\ ${ }^{1}$ Department of Mathematics, Comilla University, Cumilla, Bangladesh \\ ${ }^{2}$ Department of Applied Mathematics, University of Dhaka, Dhaka, Bangladesh \\ Email: marufek@yahoo.com
}

How to cite this paper: Hasan, M.M., Samad, M.A. and Hossain, M.M. (2020) Effects of Hall Current and Ohmic Heating on Non-Newtonian Fluid Flow in a Channel due to Peristaltic Wave. Applied Mathematics, 11, 292-306. https://doi.org/10.4236/am.2020.114022

Received: February 19, 2020

Accepted: April 13, 2020

Published: April 16, 2020

Copyright (๑) 2020 by author(s) and Scientific Research Publishing Inc. This work is licensed under the Creative Commons Attribution International License (CC BY 4.0).

http://creativecommons.org/licenses/by/4.0/

(c) (i) Open Access

\begin{abstract}
In this paper, the study of non-Newtonian fluid flow with heat transfer in a porous asymmetric channel due to peristaltic wave was taken out. Hall current and Ohmic heating effects were introduced in the present study. A Casson non-Newtonian constitutive model was employed for the transport of fluid. Analytical solutions were obtained for stream function, temperature and heat transfer coefficient. The coupled nonlinear equations have also been solved numerically using MATLAB software (by bvp4c function). The influences of many evolving parameters on the flow characteristic have been explained with the help of $2 \mathrm{D}$ and $3 \mathrm{D}$ plots. Again the obtained results were compared with the results available in the literature and were found in good agreement.
\end{abstract}

\section{Keywords}

Ohmic Heating, Non-Newtonian Fluid, Temperature Field

\section{Introduction}

At present, investigation regarding the peristaltic flow of non-Newtonian fluid cannot be ignored due to their vast applications in physiology and industry. This mechanism is associated with a spontaneous relaxing and compressing movement along the length of the fluid filled channel or tube. Digestive track, urine transport form kidney to bladder, blood flow in small vessels, egg movement in fallopian tube are few examples that can be seen in our body [1]. Latham [2] studied the initial pioneer work on peristaltic mechanism of viscous fluid and later we saw many studies [3] [4] [5] [6]. Again the study of heat transfer in pe- 
ristaltic flow has some vital roles in biomedical processes such as hemodialysis and blood oxygenation. This property helps in crude oil refinement, food processing, sanitary fluid transport, and noxious fluid transport in nuclear industries [7].

It is well known the industrial and physiological fluids are non-Newtonian in nature. Due to various rheological properties of non-Newtonian fluids, several constitutive relations have been suggested. Casson fluid is one of the non-Newtonian fluids which was introduced by Casson [8]. The behaviors of Human blood, honey and jelly can be represented by Casson's model [9]. Most of the time Hall effect is neglected in the Ohm's law as they have no remarkable effect for a weak magnetic field. It is mentioned that this effect is important when the magnetic field is high [10]. On the other hand, Ohmic heating (Joule heating) effect, which is the transformation between electric energy to thermal energy, arises from the applied electric field and fluid electrical resistivity. This effect is useful for a variety of applications in food industry [11]. Existing literatures witness that no proper attention is given to peristaltic motion of Casson fluid in an asymmetric channel with Hall current and Ohmic heating effects. The aim of the study is to fulfill this void. Mathematical modeling is carried out for low Reynolds number and long wave length approximations. The transformed equations have been solved analytically and numerically. The effects of various important parameters on velocity and temperature are displayed graphically and discussed. Trapping phenomena are also explained.

\section{Mathematical Formulation}

Consider the peristaltic flow of non-Newtonian fluid in a two dimensional porous asymmetric channel. We choose a stationary frame of reference $(X, Y)$ such that $X$ measured along the axis of the channel and $Y$ perpendicular to it. The channel walls $H_{1}$ and $H_{2}$ are maintained at constant temperature $T_{0}$ and $T_{1}$ respectively. Let $(U, V)$ be the velocity components in the frame. A strong magnetic field with magnitude $\bar{B}=\left(0,0, B_{0}\right)$ is applied and the Hall and Joule heating effects are taken into consideration. The induced magnetic field is neglected for small magnetic Reynolds number and also the externally applied electric field is assumed to be zero. The geometry (in Figure 1) of the upper and lower wall surfaces are assumed to be

$$
\left.\begin{array}{l}
Y=H_{1}=d_{1}+a_{1} \cos \left\{\frac{2 \pi}{\lambda}(X-c t)\right\} \\
Y=H_{2}=-d_{2}-a_{2} \cos \left\{\frac{2 \pi}{\lambda}(X-c t)+\phi\right\}
\end{array}\right\}
$$

where $a_{1}, a_{2}$ denote the waves amplitudes, $d_{1}+d_{2}$ is the channel width, $\lambda$ is the wave length, $t$ is the time, $c$ is the velocity of propagation and $\phi$ is the phase difference $(0 \leq \phi \leq \pi)$, in which $\phi=0$ corresponds to symmetric channel with waves out of phase and $\phi=\pi$ corresponds to waves in phase. Here $a_{1}, a_{2}, d_{1}, d_{2}$ and $\phi$ satisfies the condition 


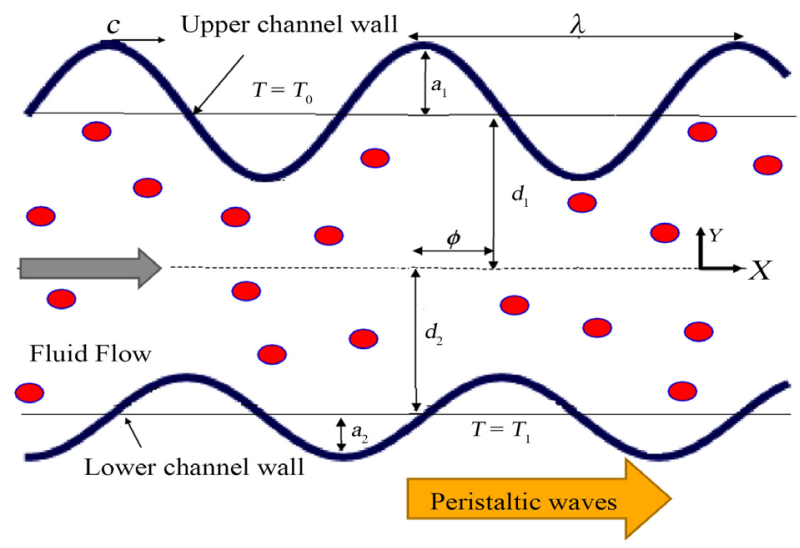

Figure 1. Geometry of the model.

$$
a_{1}^{2}+a_{2}^{2}+2 a_{1} a_{2} \cos \phi \leq\left(d_{1}+d_{2}\right)^{2}
$$

The rheological relation for non-Newtonian Casson [12] fluid is

$$
\tau_{i j}=2\left(\mu_{b}+\frac{P_{y}}{\sqrt{2 \pi}}\right) e_{i j}
$$

where $e_{i j}$ is the $(i, j)$ th component of deformation rate, $\tau_{i j}$ is the $(i, j)$ th component of the stress tensor, $\pi$ is the product of the component of deformation rate with itself and $\mu_{b}$ is the plastic dynamic viscosity. The yield stress $P_{y}$ is expressed as $P_{y}=\mu_{b} \sqrt{2 \pi} / \beta$, where $\beta$ Casson fluid parameter. For non-Newtonian Casson fluid flow $\mu=\mu_{b}+P_{y} / \sqrt{2 \pi}$ which gives $v^{\prime}=v(1+1 / \beta)$, where $v=\mu_{b} / \rho$ is the kinematic viscosity for Casson fluid. Again the yield stress $P_{y}=0$ for Newtonian case.

The equations governing the fluid motion can be written as follows

$$
\begin{gathered}
\frac{\partial U}{\partial X}+\frac{\partial V}{\partial Y}=0 . \\
\frac{\partial U}{\partial t}+U \frac{\partial U}{\partial X}+V \frac{\partial U}{\partial Y}=-\frac{1}{\rho} \frac{\partial P}{\partial X}+v\left(1+\frac{1}{\beta}\right)\left(\frac{\partial^{2} U}{\partial X^{2}}+\frac{\partial^{2} U}{\partial Y^{2}}\right) \\
+\frac{\sigma B_{\circ}^{2}}{\rho\left(1+m^{2}\right)}(m V-U)-v\left(1+\frac{1}{\beta}\right) \frac{U}{K^{\prime}} \\
\frac{\partial V}{\partial t}+U \frac{\partial V}{\partial X}+V \frac{\partial V}{\partial Y}=-\frac{1}{\rho} \frac{\partial P}{\partial Y}+v\left(1+\frac{1}{\beta}\right)\left(\frac{\partial^{2} V}{\partial X^{2}}+\frac{\partial^{2} V}{\partial Y^{2}}\right) \\
\frac{\partial T}{\rho t}+U \frac{\partial T}{\partial X}+V \frac{\partial T}{\partial Y}\left(1+m^{2}\right)(m U+V)-v\left(1+\frac{1}{\beta}\right) \frac{V}{K^{\prime}} \\
\frac{k}{\rho c_{p}}\left(\frac{\partial^{2} T}{\partial X^{2}}+\frac{\partial^{2} T}{\partial Y^{2}}\right)+\frac{v}{C_{p}}\left(1+\frac{1}{\beta}\right)\left[2\left\{\left(\frac{\partial U}{\partial X}\right)^{2}+\left(\frac{\partial V}{\partial Y}\right)^{2}\right\}+\left(\frac{\partial U}{\partial Y}+\frac{\partial V}{\partial X}\right)^{2}\right] \\
+\frac{1}{\rho c_{p}} \frac{\rho B_{\circ}^{2}}{\rho\left(1+m^{2}\right)}\left(V^{2}+U^{2}\right)
\end{gathered}
$$

Generalized Ohm's law is 


$$
\bar{J}=\sigma[\bar{E}+\bar{q} \times \bar{B}-\gamma(\bar{J} \times \bar{B})]
$$

Lorentz force is

$$
\bar{J} \times \bar{B}=\frac{\sigma B_{\circ}^{2}}{1+m^{2}}[\bar{i}(m V-U)-\bar{j}(m U+V)]
$$

Ohmic heating (Joule heating) is

$$
\frac{\bar{J} \cdot \bar{J}}{\sigma}=\frac{\sigma B_{0}^{2}}{1+m^{2}}\left(V^{2}+U^{2}\right)
$$

where $\bar{J}$ is the current density, $\bar{q}$ is the fluid velocity, $P$ is the pressure, $\gamma=1 / e n_{e}$ is the Hall factor/Hall Current, $e$ is the charge electron, $n_{e}$ is the mass of the electron, $\bar{E}$ is the electric field.

The corresponding boundary conditions are

$$
\left.\begin{array}{lll}
U=0, & T=T_{0} & \text { when } Y=H_{1} \\
U=0, & T=T_{1} & \text { when } Y=H_{2}
\end{array}\right\}
$$

The coordinates, velocity, pressure and temperature in the fixed frame $(X, Y)$ and wave frame $(x, y)$ are related by the following expression

$$
x=X-c t, y=Y, u=U-c, v=V, p(x, y)=P(X, Y, t), \bar{T}(x, y)=T(X, Y, t)
$$

where $u, v, p, \bar{T}$ are the velocity components, pressure and temperature in the wave frame respectively.

Now we introduce the following dimensionless quantities

$$
\left.\begin{array}{l}
x^{\prime}=\frac{x}{\lambda}, \quad y^{\prime}=\frac{y}{d_{1}}, \quad u^{\prime}=\frac{u}{c}, \quad v^{\prime}=\frac{v}{c \delta}, \quad t^{\prime}=\frac{c t}{\lambda}, \quad p^{\prime}=\frac{p d_{1}^{2}}{\lambda c \mu_{b}} \\
\delta=\frac{d_{1}}{\lambda}, h_{1}=\frac{H_{1}}{d_{1}}, \quad h_{2}=\frac{H_{2}}{d_{1}}, \quad d=\frac{d_{2}}{d_{1}}, \quad a=\frac{a_{1}}{d_{1}}, \quad b=\frac{a_{2}}{d_{1}}, \quad \theta=\frac{\bar{T}-\bar{T}_{0}}{\bar{T}_{1}-\bar{T}_{0}}
\end{array}\right\}
$$

The governing Equations (4)-(7) under the assumptions of long wave length and low Reynolds number in terms of stream function $\psi$ (dropping the das symbols) become

$$
\begin{gathered}
\frac{\partial p}{\partial x}=\left(1+\frac{1}{\beta}\right)\left[\frac{\partial^{3} \psi}{\partial y^{3}}-\alpha^{2}\left(\frac{\partial \psi}{\partial y}+1\right)\right] \\
\frac{\partial p}{\partial y}=0 \\
\theta^{\prime \prime}+\left(1+\frac{1}{\beta}\right) B r\left(\frac{\partial^{2} \psi}{\partial y^{2}}\right)^{2}+B r \frac{M^{2}}{1+m^{2}}\left(\frac{\partial \psi}{\partial y}+1\right)^{2}=0
\end{gathered}
$$

The dimensionless boundary conditions become

$$
\left.\begin{array}{l}
\psi=\frac{F}{2}, \quad \frac{\partial \psi}{\partial y}=-1, \theta=0 \quad \text { when } y=h_{1} \\
\psi=\frac{-F}{2}, \frac{\partial \psi}{\partial y}=-1, \theta=1 \quad \text { when } y=h_{2}
\end{array}\right\}
$$

where $\alpha^{2}=\frac{M^{2}}{\left(1+m^{2}\right)(1+1 / \beta)}+\frac{1}{K}, M=\sqrt{\frac{\sigma}{\mu_{b}}} B_{0} d_{1}$ is the magnetic field para- 
meter, $K=\frac{K^{\prime}}{d_{1}^{2}}$ is the permeability parameter, $F$ is the volume flow rate in the wave frame, $\beta=\mu_{b} \sqrt{2 \pi} / P_{y}$ is the Casson fluid parameter, $m=\sigma \gamma B_{0}$ is the Hall parameter, $\operatorname{Pr}=\frac{\rho v C_{p}}{k}$ is Prandtl number, $E c=\frac{c^{2}}{C_{p}\left(\bar{T}_{1}-\bar{T}_{0}\right)}$ is the Eckert number and $\mathrm{Br}=\mathrm{Pr} \cdot E \mathrm{C}$ is the Brinkman number.

\section{Exact Solution}

Equation (15) gives that $p \neq p(y)$. Eliminating the pressure terms from (14) we get

$$
\frac{\partial^{4} \psi}{\partial y^{4}}-\alpha^{2} \frac{\partial^{2} \psi}{\partial y^{2}}=0
$$

Solving Equations (18) and (16) with the boundary conditions (17), the stream function and temperature are obtained as

$$
\begin{gathered}
\psi=B_{1}+B_{2} y+B_{3} \cosh (\alpha y)+B_{4} \sinh (\alpha y) \\
\theta=-F_{1} y^{2}-F_{2} \cosh (2 \alpha y)-F_{3} \sinh (2 \alpha y)-F_{4} \sinh (\alpha y) \\
-F_{5} \cosh (\alpha y)-B_{5} y-B_{6}
\end{gathered}
$$

where the constants involved in the solutions are given in the appendix.

The dimensionless mean flow rate $Q^{\prime}$ in the laboratory frame is related to the dimensionless mean flow rate $F$ in the wave frame by

$$
Q^{\prime}=F+1+d
$$

in which

$$
F=\int_{h_{2}}^{h_{1}} u \mathrm{~d} y
$$

Also note that $h_{1}$ and $h_{2}$ represent the dimensionless forms of the peristaltic walls

$$
\left.\begin{array}{l}
h_{1}=1+a \cos (2 \pi x) \\
h_{2}=-d-b \cos (2 \pi x+\phi)
\end{array}\right\}
$$

Again the heat transfer coefficient at the upper wall $\left(y=h_{1}\right)$ is

$$
Z_{1}=h_{1 x} \theta^{\prime}
$$

\section{Numerical Solution}

The dimensionless equations have again been solved numerically for different values of model parameters with another code written in MATLAB (bvp4c function). It is a finite difference code that implements a collocation formula. Numerical computations have been carried out for magnetic field parameter $(M)$, Hall parameter $(m)$, Casson fluid parameter $(\beta)$, permeability parameter $(K)$ and Brinkman number $(B r)$ at fixed values of $a=0.3, b=0.5, d=1.3, \phi=\pi / 3, F=-1.5$ and $x=0$. The software ORIGIN has been used to show the numerical results 
graphically. Plots $2 \mathrm{D}$ and $3 \mathrm{D}$ are sketched to understand and explain the varying activities of model parameters in a better way.

\section{Results and Discussions}

The effect of magnetic field parameter $M$ on velocity field is sketched in Figure 2. Here we see that the velocity decreases with an increase in $M$. This is due to the fact that the applied magnetic field produces a Lorentz force to the flow and this force reduces the velocity. Figure 3 is plotted to see the velocity profiles for different permeability parameter $K$. There is a grow in the velocity of fluid near the central part of the channel when we increase in $K$. This is due to the fact that large $K$ provides less resistance to the flow. From Figure 4 , it is clear that $\beta$ has an increasing effect on velocity field. Large $\beta$ accelerates the fluid flow. The magnitude of the velocity $u$ increases with increasing Hall parameter $m$ as seen in Figure 5. The fact is that the effective conductivity $\sigma /\left(1+m^{2}\right)$, existed in the momentum equation, diminishes when $m$ increased which consequently reduces the magnetic Lorentz force. Therefore velocity increases. Figure 6 shows that the velocity increases with an increase in flow rate $F$.
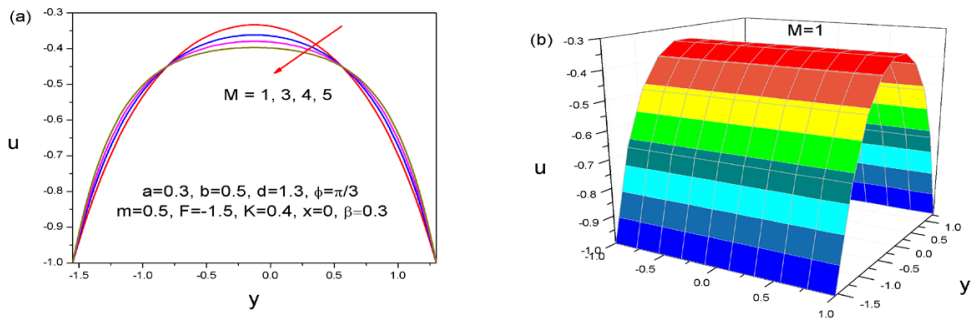

Figure 2. Velocity profiles for different values of magnetic field parameter $M$ (a) 2D and (b) 3D.
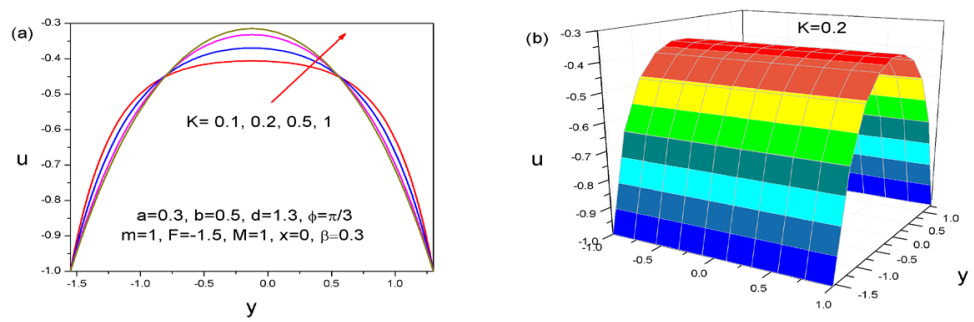

Figure 3. Velocity profiles for different values of permeability parameter $K$ (a) $2 \mathrm{D}$ and (b) $3 \mathrm{D}$.
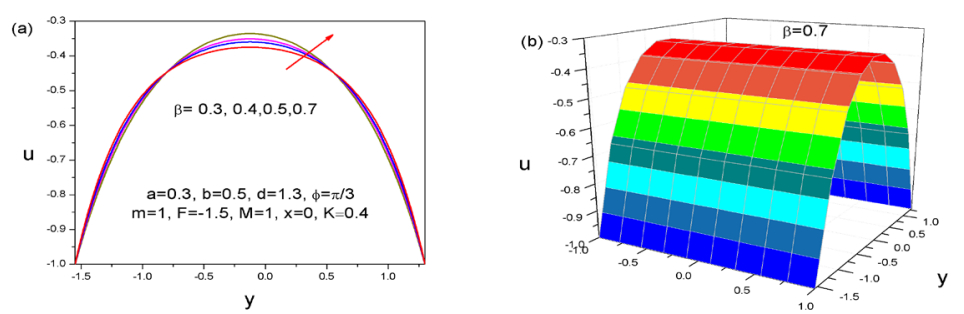

Figure 4. Velocity profiles for different values of Casson fluid parameter $\beta$ (a) $2 \mathrm{D}$ and (b) $3 \mathrm{D}$. 

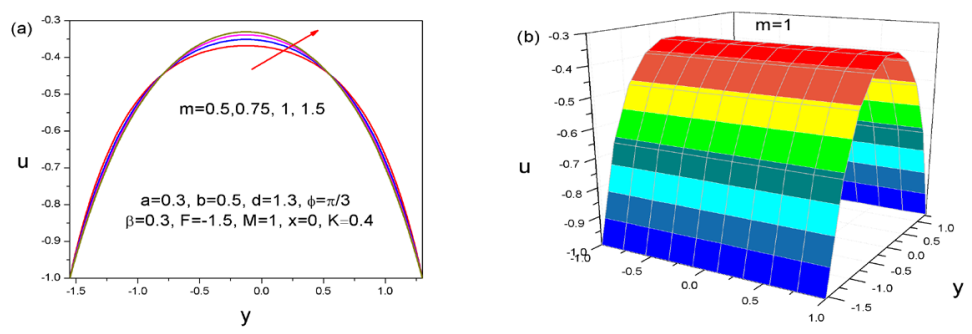

Figure 5. Velocity profiles for different values of Hall parameter $m$ (a) $2 \mathrm{D}$ and (b) 3D.
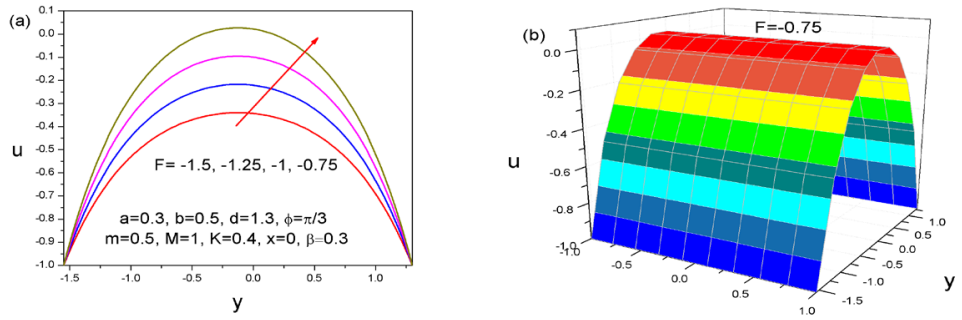

Figure 6. Velocity profiles for different values of flow rate $F(a) 2 D$ and (b) 3D.

Figures 7-11 show the temperature profile $\theta$ for different values of $M, K, \beta, m$ and $B r$. Here we see that temperature profiles are almost parabolic in character and higher temperature is seen near the centre of the channel. Figure 7 depicts that as $M$ increased, the temperature profile increases. The behavior of $K$ on temperature profile is seen in Figure 8. Here we notice that temperature increases for large values of $K$. The influence of Casson fluid parameter $\beta$ on temperature profiles is illustrated in Figure 9. It reveals that temperature profile decreases with an increase in $\beta$. Figure 10 indicates that temperature decreases as $m$ increased. Joule heating is proportional to $1 /\left(1+m^{2}\right)$. Large $m$ results a reduction in Joule heating. For this reason, temperature decreases when $m$ is increased. The effect of Brinkman number $B r$ on temperature profiles is displayed in Figure 11. Here the temperature $\theta$ increases gradually with an increase in $B r$. Brinkman number $B r(=P r \cdot E c)$ represents the viscous effects which are due to energy production and thus heats up the fluid.

In order to see the job of Ohmic (Joule) heating effect we have resolved the heat equation after neglecting the Ohmic heating term and then investigative the magnetic field parameter $M$ and Hall parameter $m$ effects on temperature field through graphs. Figure 12 and Figure 13 show that temperature decreases for increasing $M$ and increases for large $m$. From the above discussion it is clear that when we neglect the Ohmic heating term, the behaviors of $M$ and $m$ on temperature field are quite opposite.

Trapping, the procedure of contours of streamlines, is an attractive characteristic for peristaltic flow. To investigate the effects of $M, m$ and $\phi$ on streamlines, we have plotted Figures 14-16. Here we notice that the size of trapped bolus (trapped bolus is a bolus of fluid which is enclosed by streamlines) decreases for large values of magnetic field parameter $M$ as seen in Figure 14 (among them (a), (c) for symmetric channel and (b), (d) for asymmetric channel). On the oth- 
er hand, opposite behavior is observed for Hall parameter $m$. Figure 16 indicates the impact of phase difference $\phi$ on streamlines. It is clear that the bolus travels upwards and decreases in size as $\phi$ gets large. Also the trapping vanishes when $\phi$ reaches to $\pi$.
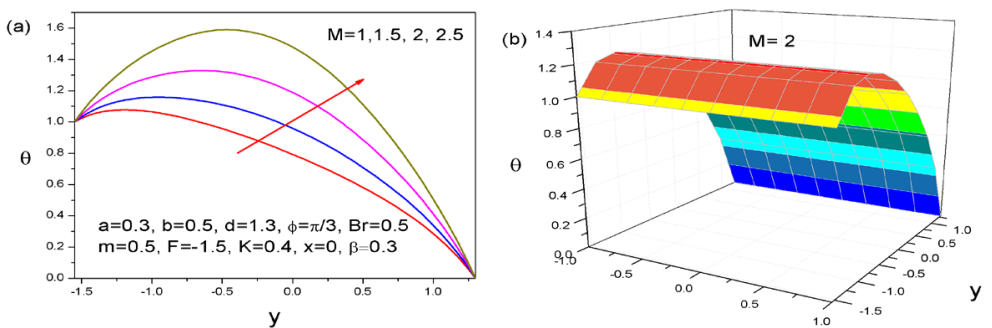

Figure 7. Temperature profiles for different values of magnetic field parameter $M$ (a) 2D and (b) 3D.
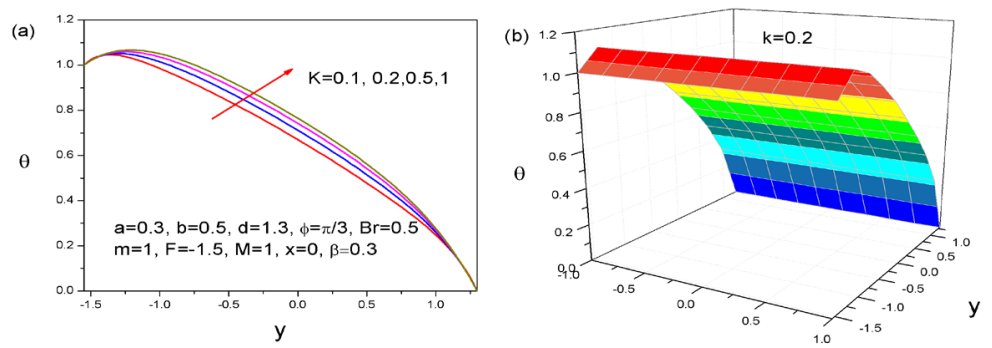

Figure 8. Temperature profiles for different values of permeability parameter $K(\mathrm{a}) 2 \mathrm{D}$ and (b) 3D.
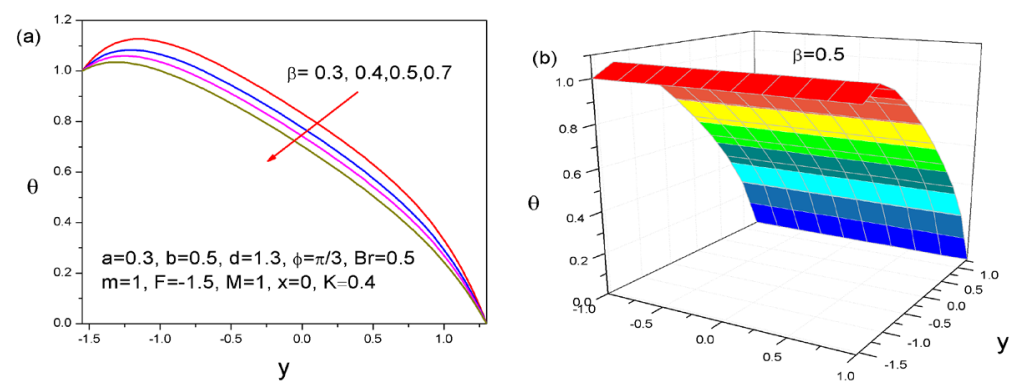

Figure 9. Temperature profiles for different values of Casson fluid parameter $\beta$ (a) 2D and (b) 3D.
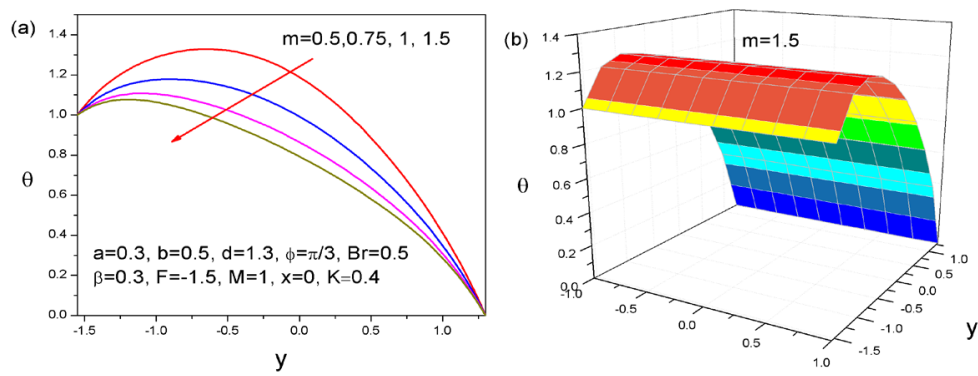

Figure 10. Temperature profiles for different values of Hall parameter $m$ (a) 2D and (b) 3D. 

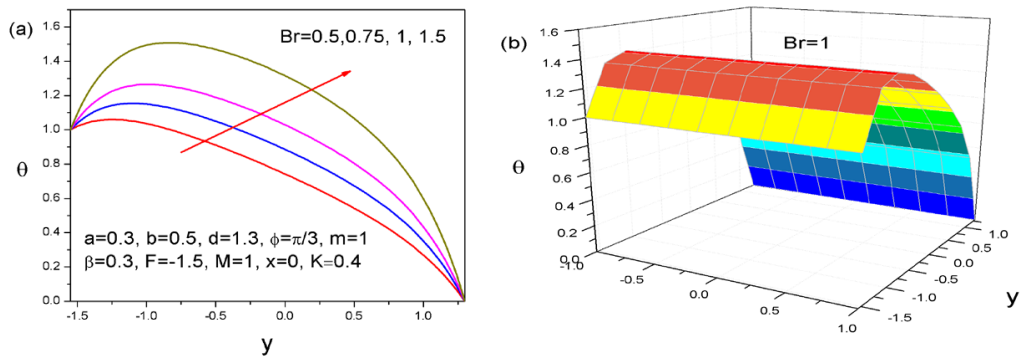

Figure 11. Temperature profiles for different values of Brinkman number $B r(a) 2 D$ and (b) 3D.

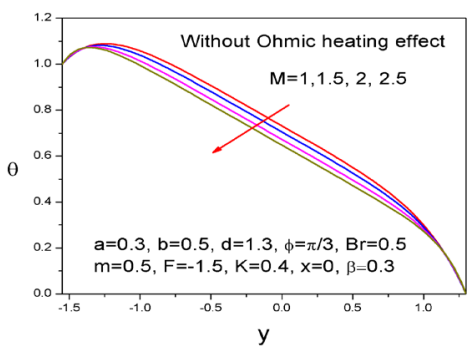

Figure 12. Temperature profiles for different values of magnetic field parameter $M$.

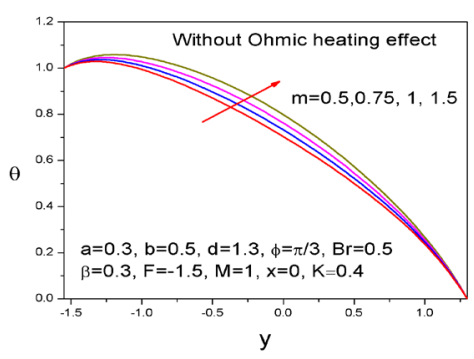

Figure 13. Temperature profiles for different values of Hall parameter $m$.

To verify the accuracy of numerical results, the present study is compared with the previous study of Hayat et al. [13]. We have considered the same parameter values (Newtonian case and in absence of Ohmic heating) to do it. These comparisons are given in Figure 17, which are found in very good agreement. Again Table 1 gives a comparison of heat transfer coefficient $Z_{1}$ at upper wall for different values of $K$ (Newtonian case and in absence of Ohmic heating).

Table 1. Comparison of heat transfer coefficient $Z_{1}$ at upper wall for different values of $K$.

\begin{tabular}{|c|c|c|c|c|}
\hline \multicolumn{5}{|c|}{$a=0.5, b=0.6, d=1.5, M=2, \phi=\pi / 4, F=-2, B r=3$} \\
\hline \multirow{2}{*}{$x$} & Present & Hayat et al. [13] & Present & Hayat et al. [13] \\
\hline & \multicolumn{2}{|c|}{$K=0.1$} & \multicolumn{2}{|c|}{$K=1$} \\
\hline 0.1 & 2.31567 & 2.31697 & 1.98257 & 1.98138 \\
\hline 0.2 & 1.96850 & 1.96953 & 1.86659 & 1.86796 \\
\hline 0.3 & 2.01043 & 2.01085 & 1.98089 & 1.98128 \\
\hline
\end{tabular}




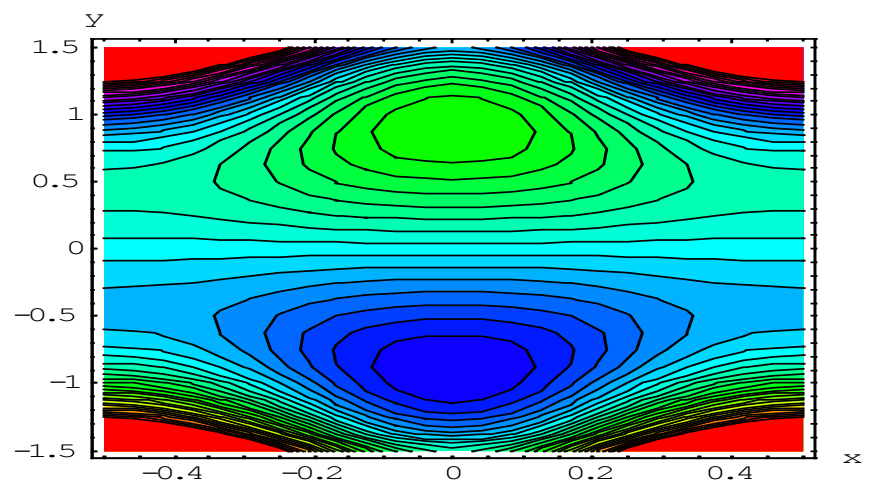

(a) $M=1, \phi=0$

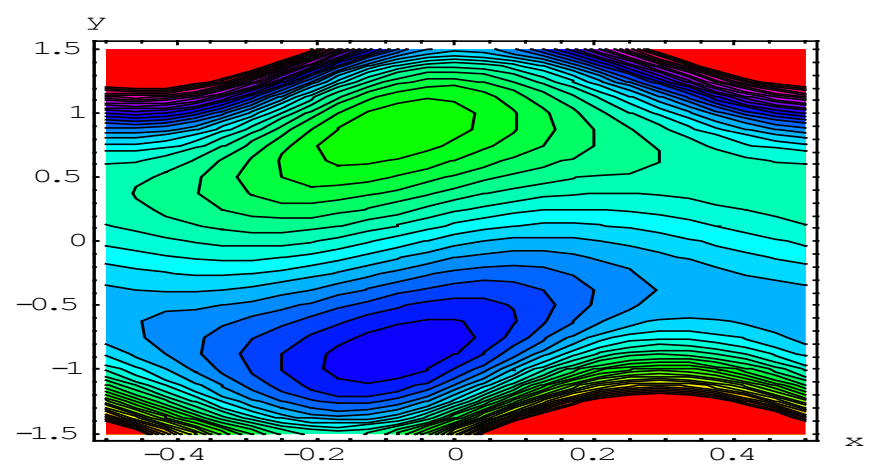

(b) $M=1, \phi=\pi / 3$

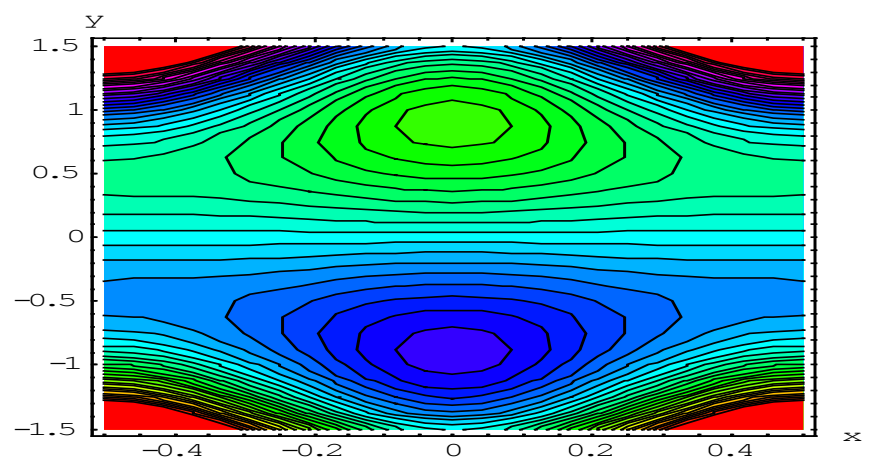

(c) $M=3, \phi=0$

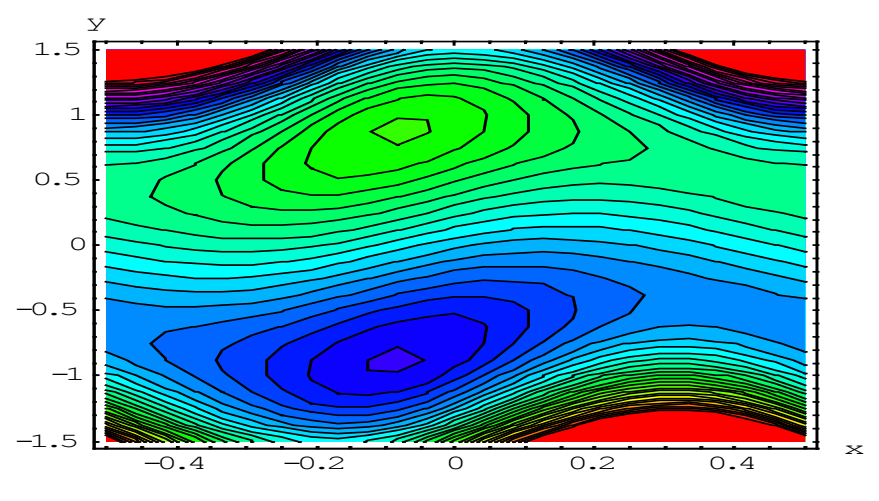

(d) $M=3, \phi=\pi / 3$

Figure 14. Streamline patterns for different values of magnetic field parameter $M$. 


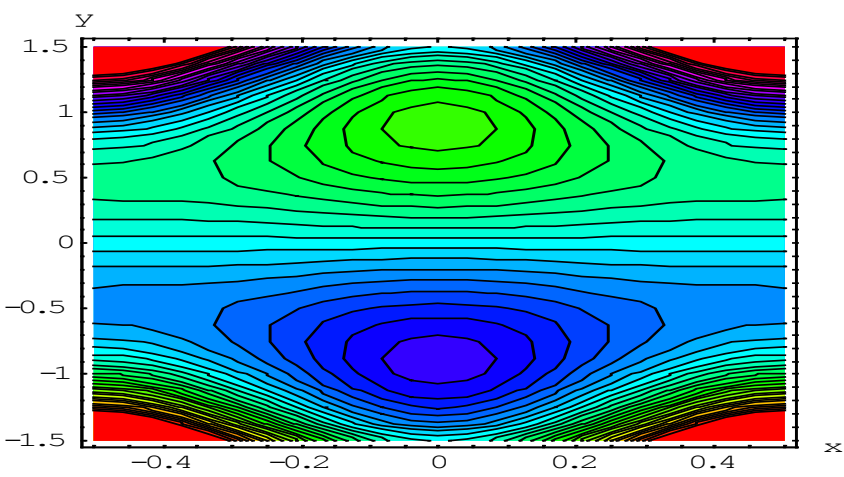

(a) $m=0.3, \phi=0$

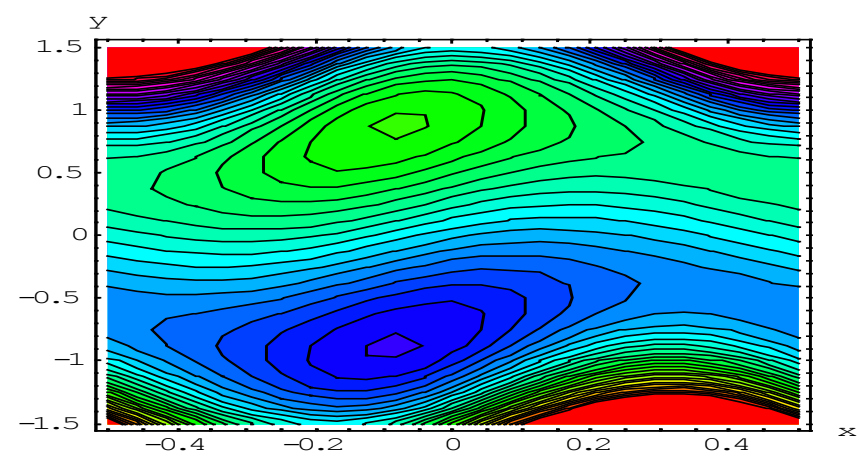

(b) $m=0.3, \phi=\pi / 3$

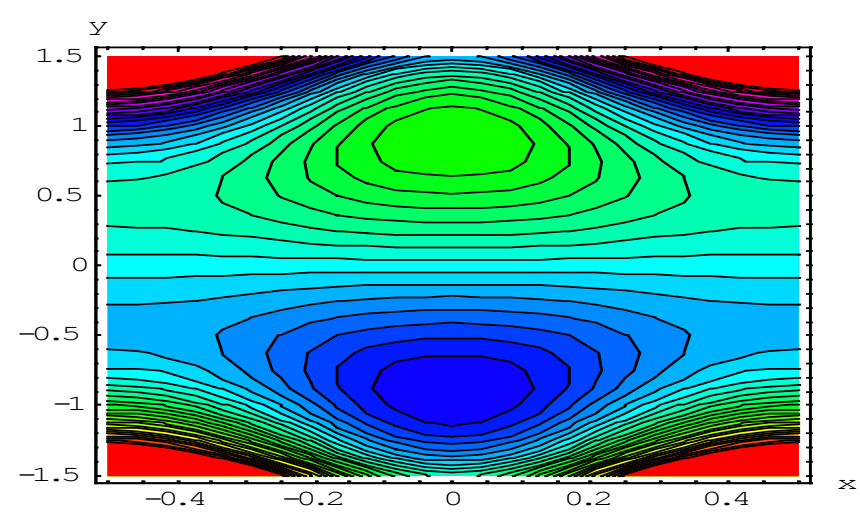

(c) $m=1, \phi=0$

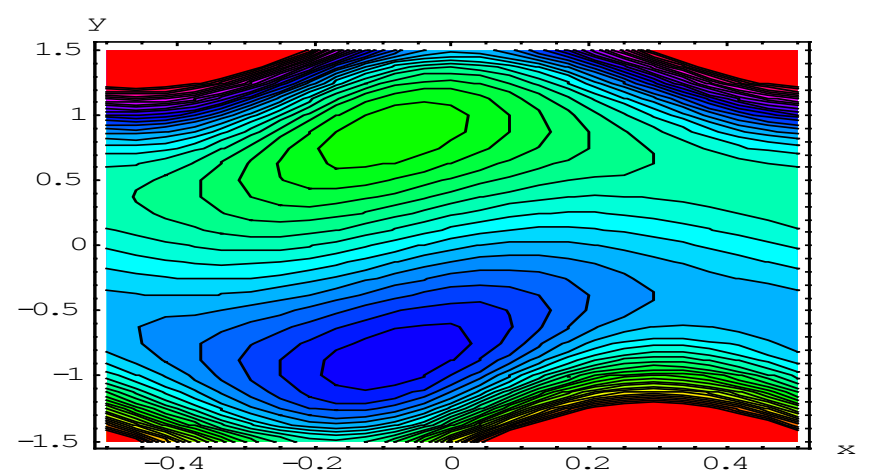

(d) $m=1, \phi=\pi / 3$

Figure 15. Streamline patterns for different values of Hall parameter $m$. 


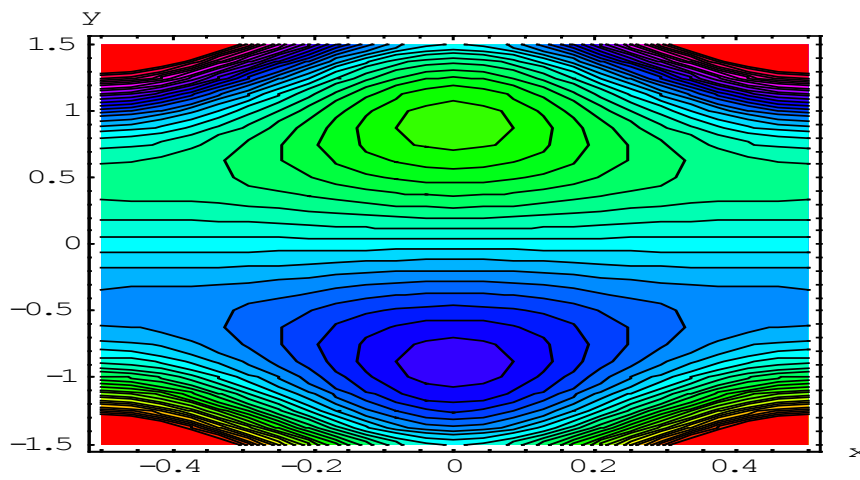

(a) $\phi=0$

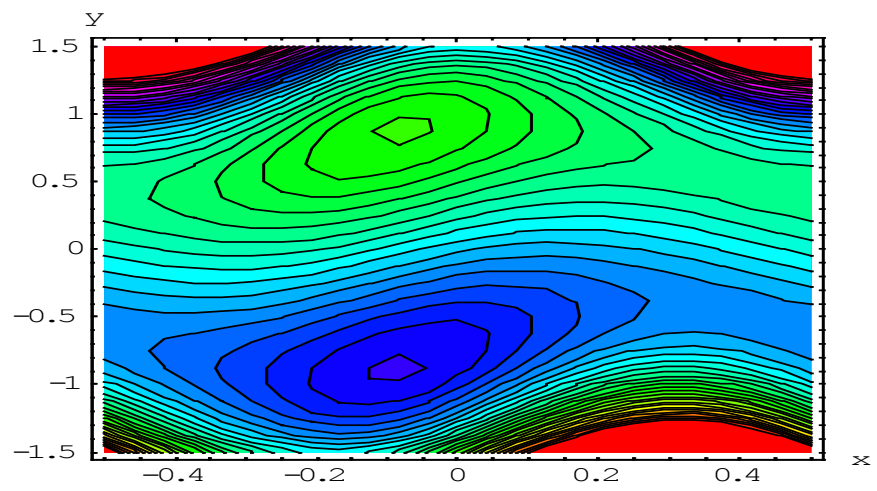

(b) $\phi=\pi / 3$

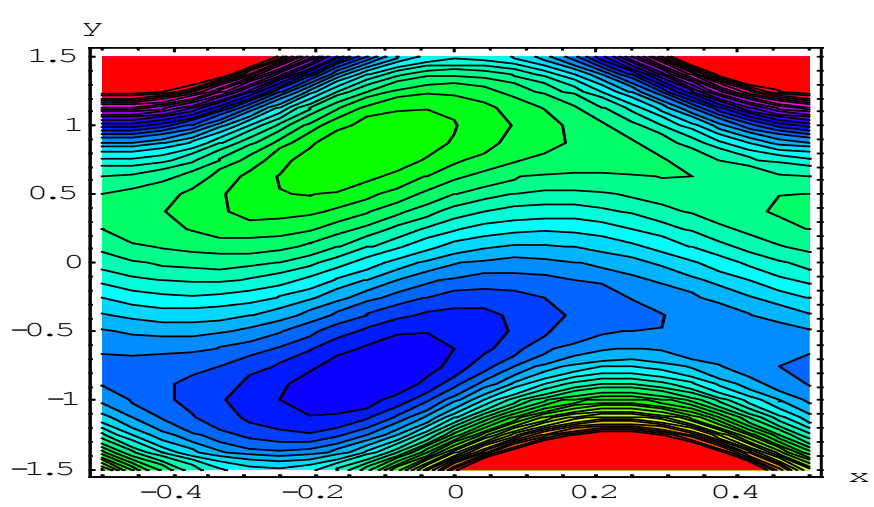

(c) $\phi=\pi / 2$

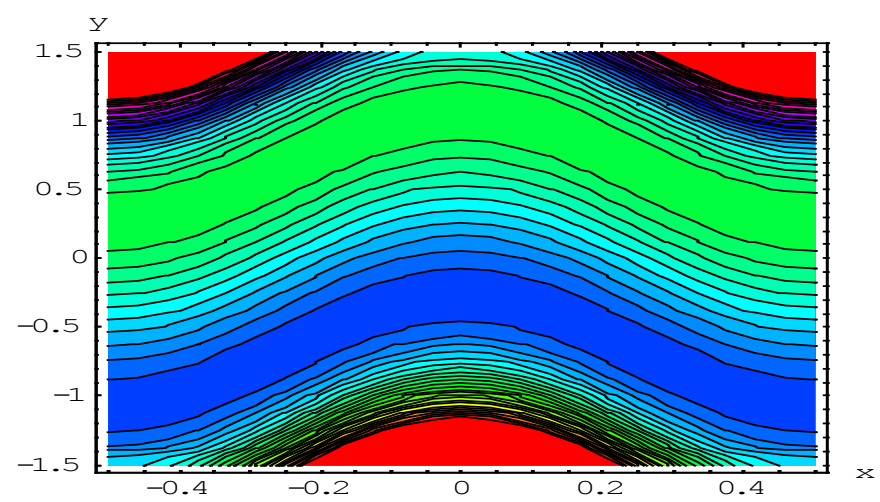

(d) $\phi=\pi$

Figure 16. Streamline patterns for different values of phase difference $\phi$. 


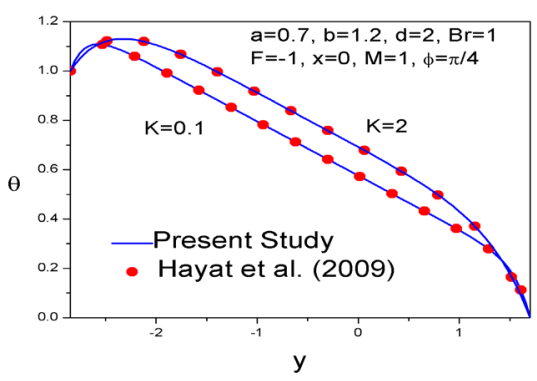

Figure 17. Comparison of Temperature profiles.

\section{Conclusions}

This study is presented on the peristaltic motion of non-Newtonian fluid in a porous asymmetric channel with Hall current and Ohmic heating effects. A Casson non-Newtonian constitutive model was employed for this study. Both analytic and numerical solutions have been obtained. The effects of different parameters on flow characteristics are shown by using $2 \mathrm{D}$ and $3 \mathrm{D}$ plots. The main outcomes of the study are:

1) Magnetic field parameter $M$ has decreasing effect but Hall parameter $m$ has an increasing impact on velocity profile.

2) The fluid becomes less viscous when we increase $\beta$.

3) Temperature decreases when $m$ increases and increases when $M$ and $B r$ increase.

4) The activities of $M$ and $m$ on temperature field are quite reverse when we neglect the Ohmic heating term.

5) The size of trapped bolus decreases for large values of phase difference $\phi$ and trapping vanishes when $\phi=\pi$.

\section{Acknowledgements}

The authors are thankful to the referees for their useful suggestions.

\section{Conflicts of Interest}

The authors declare no conflicts of interest regarding the publication of this paper.

\section{References}

[1] Reddy, K.V. and Reddy, M.G. (2014) Velocity Slip and Joule Heating Effects on MHD Peristaltic Flow in a Porous Medium. International Journal of Applied Mathematics and Mechanics, 2, 126-138.

[2] Latham, T.W. (1966) Fluid Motion in a Peristaltic Pump. MS Thesis, MIT, Cambridge.

[3] Ahmed, B., Javed, T. and Ali, N. (2018) Numerical Study at Moderate Reynolds Number of Peristaltic Flow of Micropolar Fluid through a Porous-Saturated Channel in Magnetic Field. AIP Advances, 8, Article ID: 015319. https://doi.org/10.1063/1.5019380

[4] Abd-Alla, A.M., Abo-Dahab, S.M. and Al-Simery, R.D. (2013) Effect of Rotation on 
Peristaltic Flow of a Micropolar Fluid through a Porous Medium with an External Magnetic Field. Journal of Magnetism and Magnetic Materials, 348, 33-43. https://doi.org/10.1016/j.jmmm.2013.06.030

[5] Misra, J.C. and Sinha, A. (2013) Effect of Thermal Radiation on MHD Flow of Blood and Heat Transfer in a Permeable Capillary in Stretching Motion. Heat Mass Transfer, 49, 617-628. https://doi.org/10.1007/s00231-012-1107-6

[6] Elangovan, K. and Selvaraj, K. (2017) MHD Peristaltic Flow of Blood through Porous Medium with Slip Effect in the Presence of Body Acceleration. World Journal of Modelling and Simulation, 13, 151-160.

[7] Pandey, S.K. and Tripathi, D. (2011) A Mathematical Model for Swallowing of Concentrated Fluids in Oesophagus. Applied Bionics and Biomechanics, 8, 309-321. https://doi.org/10.1155/2011/782414

[8] Casson, N. (1959) Rheology of Disperse Systems. Pergamon Press, London, 84.

[9] Blair, S., William, G. and Spanner, D.C. (1974) Introduction to Biorheology by GW Scott Blair, Chapter XII on Botanical Aspects by DC Spanner. Elsevier Science Ltd., Amsterdam.

[10] Hayat, T., Iqbal, M., Yasmin, H. and Alsaadi, F. (2014) Hall Effects on Peristaltic Flow of Couple Stress Fluid in an Inclined Asymmetric Channel. International Journal of Biomathematics, 7, 1-34. https://doi.org/10.1142/S1793524514500570

[11] Munir, A.F., Tasawar, H. and Bashir, A. (2014) Peristaltic Flow in an Asymmetric Channel with Convective Boundary Conditions and Joule Heating. Journal of Central South University, 21, 1411-1416. https://doi.org/10.1007/s11771-014-2079-0

[12] Eldabe, N.T.M., Saddeck, G. and El-Sayed, A.F. (2001) Heat Transfer of MHD Non-Newtonian Casson Fluid Flow between Two Rotating Cylinders. Mechanics and Mechanical Engineering, 5, 237-251.

[13] Hayat, T. Qureshi, M.U. and Hussain, Q. (2009) Effect of Heat Transfer on Peristaltic Flow of an Electrically Conducting Fluid in a Porous Space. Applied Mathematical Modelling, 33, 1862-1873. https://doi.org/10.1016/j.apm.2008.03.024 


\section{Appendix}

The values involved in the solution expressions are presented below.

\begin{tabular}{ccc}
\hline$G_{1}=\cosh \frac{\alpha}{2}\left(h_{1}-h_{2}\right)$ & $D_{3}=\sinh \left(\alpha h_{2}\right)-\sinh \left(\alpha h_{1}\right)$ & $F_{2}=E_{4} E_{2}$ \\
$G_{2}=\sinh \frac{\alpha}{2}\left(h_{1}-h_{2}\right)$ & $D_{4}=\cosh \left(\alpha h_{2}\right)-\cosh \left(\alpha h_{1}\right)$ & $F_{3}=E_{4} E_{3}$ \\
$B_{1}=\frac{-\left(h_{1}+h_{2}\right)\left[F \alpha G_{1}+2 G_{2}\right]}{2\left(h_{1}-h_{2}\right) \alpha G_{1}-4 G_{2}}$ & $E_{1}=\left(B_{3}^{2}-B_{4}^{2}\right) / 2$ & $F_{4}=E_{5} B_{3}$ \\
$B_{2}=\frac{F \alpha G_{1}+2 G_{2}}{\left(h_{1}-h_{2}\right) \alpha G_{1}-2 G_{2}}$ & $E_{2}=\frac{B_{3}^{2}+B_{4}^{2}}{2}$ & $F_{5}=E_{5} B_{4}$ \\
$B_{3}=\frac{\left(h_{1}-h_{2}+F\right) \sinh \frac{\alpha}{2}\left(h_{1}+h_{2}\right)}{\left(h_{1}-h_{2}\right) \alpha G_{1}-2 G_{2}}$ & $E_{3}=B_{3} B_{4}$ & $P_{1}=B r(1+1 / \beta)$ \\
$B_{4}=\frac{\left(h_{1}-h_{2}+F\right) \cosh \frac{\alpha}{2}\left(h_{1}+h_{2}\right)}{\left(h_{1}-h_{2}\right) \alpha G_{1}-2 G_{2}}$ & $E_{4}=\frac{P_{1} \alpha^{2}+P_{2}}{4}$ & $P_{2}=B r \frac{M^{2}}{1+m^{2}}$ \\
$D_{1}=\cosh \left(2 \alpha h_{2}\right)-\cosh \left(2 \alpha h_{1}\right)$ & $E_{5}=2 P_{2} P_{3} / \alpha$ & $P_{3}=1+B_{2}$ \\
$D_{2}=\sinh \left(2 \alpha h_{2}\right)-\sinh \left(2 \alpha h_{1}\right)$ & $F_{1}=\left(\left(P_{1} \alpha^{2}-P_{2}\right) E_{1} \alpha^{2}+P_{1} P_{3}^{2}\right) / 2$ & \\
$B_{5}=\left[1+F_{1}\left(h_{2}^{2}-h_{1}^{2}\right)+F_{2} D_{1}+F_{3} D_{2}+F_{4} D_{3}+F_{5} D_{4}\right] /\left(h_{2}-h_{1}\right)$ & \\
$B_{6}=-B_{5} h_{1}-F_{1} h_{1}^{2}-F_{2} \cosh \left(2 \alpha h_{1}\right)-F_{3} \sinh \left(2 \alpha h_{1}\right)-F_{4} \sinh \left(\alpha h_{1}\right)-F_{5} \cosh \left(\alpha h_{1}\right)$
\end{tabular}

